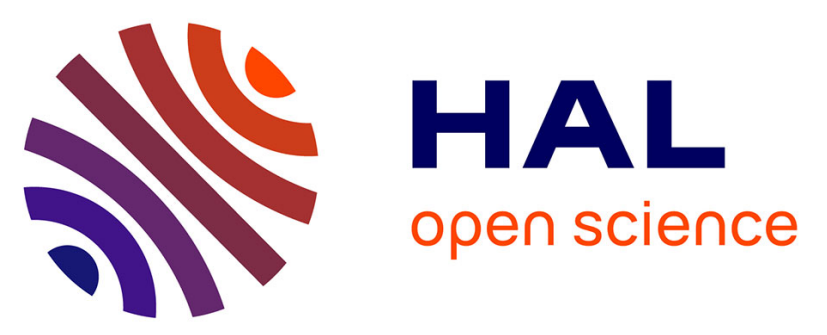

\title{
Non-invasive evaluation of retinal vascular remodeling and hypertrophy in humans: intricate effect of ageing, blood pressure and glycaemia
}

\author{
Antonio Gallo, Thomas Dietenbeck, Alain Giron, Michel Paques, Nadjia \\ Kachenoura, Xavier Girerd
}

\section{To cite this version:}

Antonio Gallo, Thomas Dietenbeck, Alain Giron, Michel Paques, Nadjia Kachenoura, et al.. Noninvasive evaluation of retinal vascular remodeling and hypertrophy in humans: intricate effect of ageing, blood pressure and glycaemia. Clinical Research in Cardiology, In press, 10.1007/s00392-02001680-3 . hal-02880928

\section{HAL Id: hal-02880928 \\ https://hal.sorbonne-universite.fr/hal-02880928}

Submitted on 25 Jun 2020

HAL is a multi-disciplinary open access archive for the deposit and dissemination of scientific research documents, whether they are published or not. The documents may come from teaching and research institutions in France or abroad, or from public or private research centers.
L'archive ouverte pluridisciplinaire HAL, est destinée au dépôt et à la diffusion de documents scientifiques de niveau recherche, publiés ou non, émanant des établissements d'enseignement et de recherche français ou étrangers, des laboratoires publics ou privés. 


\title{
Non-invasive evaluation of retinal vascular remodeling and hypertrophy in humans: intricated effect of
} ageing, blood pressure and glycaemia.

\author{
Antonio Gallo ${ }^{1,2}$, Thomas Dietenbeck ${ }^{2}$, Alain Giron ${ }^{2}$, Michel Paques $^{3}$, Nadjia Kachenoura ${ }^{2}$, Xavier Girerd ${ }^{1}$ \\ ${ }^{1}$ Cardiovascular Prevention Unit, Department of Endocrinology and Metabolism, University Hospital Pitié- \\ Salpêtrière, APHP.6 Sorbonne University, Paris, France \\ ${ }^{2}$ Sorbonne Université, INSERM 1146, CNRS 7371, Laboratoire d’Imagerie Biomédicale, 75006, Paris, France \\ ${ }^{3}$ Département d'ophtalmologie, Hôpital des Quinze-Vingts, Centre d'investigation Clinique INSERM-DHOS \\ 1423, Institut de la Vision, Sorbonne Universités, CNRS, 75012 Paris, France.
}

\section{Corresponding author:}

Antonio Gallo, Service d'Endocrinologie métabolisme et prévention cardiovasculaire, Unité Fonctionnelle d'Aphérèse, Institut E3M et IHU cardiométabolique, Hôpital Pitié Salpêtrière, Paris, France.

Email: antonio.gallo@aphp.fr $\quad$ Tel: + 33-1-42-17-57-74 $\quad$ Fax: + (33) 1-42-17-78-32

\section{Acknowledgements}

The authors want to thank Dr Alessandro Mattina and Mrs Caroline Kanagasabapathy for their help in the image acquisition; Dr David Rosenbaum for his scientific support; We gratefully acknowledge the expert technical assistance of Mr Nicolas Chateau, Mr Laurent Vabre and Ms Martine Durand.

\begin{abstract}
:
Background. Ageing, hypertension and diabetes have an intricated effect on microvascular structure. In the retina, the respective contribution of remodeling and hypertrophy in such process is still unclear. We aimed at disentangling age, blood pressure and glycaemia effects on retinal microcirculation using the non-invasive adaptive optics ophthalmoscopy (AOO).

Methods. We included 429 subjects, distributed into 4 groups according to normal (nBP) or high blood pressure (hBP) and/or normal (nGly) or high fasting glycaemia (hGly). The nBP/nGly group was stratified in age tertiles to isolate effect of ageing. AOO was used to measure arteriolar wall thickness (WT, $\mu \mathrm{m})$, arteriolar (aID, $\mu \mathrm{m})$ and venular internal diameter $(\mathrm{vID}, \mu \mathrm{m})$ and calculate arteriolar wall-to-lumen ratio (WLR), wall cross-sectional area $\left(\mathrm{WCSA}, \mu \mathrm{m}^{2}\right)$. One-way ANOVA for parametric variables and Kruskal-Wallis test for non-parametric variables were used for comparison among groups. A multivariate regression analysis including age, gender, BP, hGly and antihypertensive treatment was performed to calculate independent predictors of retinal remodeling.
\end{abstract}

Results. WT was increased with ageing (tertile1: $22.5 \pm 3.2$, tertile2: $24.2 \pm 3.5$, tertile $3: 25.2 \pm 3.8, \mathrm{p}=0.001$ ) and BP (hBP: $25.2 \pm 4.1$ vs nBP: 23.9 $\pm 3.7, \mathrm{p}=0.003$ ). aID decreased with BP (hBP: $90.2 \pm 13.4$ vs nBP: 93.6 \pm 11.6 , $\mathrm{p}=0.013$ ) and increased with glycaemia (hGly: $97.7 \pm 12.5$ vs nGly: $93.6 \pm 11.6, p=0.002$ ). A multivariate analysis showed independent association of hBP with WLR and CV aID; hGly with WCSA; ageing with WLR and WCSA.

Conclusions. AOO non-invasively identifies retinal structural changes in human confirming that microvascular remodeling is exclusively related to hypertension, whereas vascular growth is related to ageing and hyperglycaemia.

Keywords: aging, hyperglycaemia, hypertension/high blood pressure, microcirculation, adaptive optics, retina 


\section{Introduction}

Ageing, hypertension (HT) and Type 2 Diabetes (T2D) are the main driving factors of macro-and micro-vascular changes $(1,2)$. While the effects of hypertension and diabetes on microcirculation have been well studied in invitro and in-vivo studies, the role of ageing is still not well defined $(3,4)$. In the retinal microcirculation, progressive changes going from vasospasm to microaneurysms leading to retinal ischemia, follow the progression of HT and have been related with an increased risk of cardiovascular and cerebrovascular disease (5-8). Also, arteriolar widening and capillary rarefaction as well as expression of the impaired nitric oxide (NO)release and autonomic dysfunction induced by insulin-resistance, are implicated in T2D onset (9-11). In ageing, small clinical trials have shown conflicting results: despite a decreased vessel density, no changes in arteriolar calibre have been found, suggesting that either micro-vascular function might not be influenced by ageing or that underlying changes could not be identified with the current techniques (12-14).

Adaptive optics ophthalmoscopy (AOO) allows a near-histological evaluation of retinal microvasculature with a resolution of $1.5 \mu \mathrm{m}$. This technique has been proven highly reproducible and well correlated with gold standard measurements (15). Since it provides a direct measure of arteriolar wall, it may help clarifying the structural changes in cardiometabolic conditions (16).

Previous studies have shown an inward eutrophic remodeling in HT, characterised by an arteriolar vasoconstriction and a relative wall thickening, determined by an increase in media-to-lumen and/or wall-tolumen ratio (WLR) while wall cross-sectional area (WCSA) remains unchanged $(17,18)$. In diabetes, however, an hypertrophic remodeling, with a higher WLR associated with an increased WCSA has been found $(2,19)$. Whether vascular remodeling takes place together with hypertrophy in diabetes is still unclear: the concomitant presence of T2D and HT has been related with an hypertrophic remodeling, suggesting that changes in the vascular mass might take place on the top of structural modifications within the vessel wall, and these could be associated with a higher cardiovascular risk (19).

The aims of this study were: 1) to explore retinal microvascular structural changes related to ageing and conditions of high blood pressure (hBP) and/or glycaemia (hGly) using an extensive evaluation of vascular structural components with the non-invasive and high resolution AOO, 2) to disentangle age, blood pressure and glycaemia effects on retinal microcirculation in a cohort of 429 subjects.

\section{Methods}

\section{Study population}

In this retrospective study, 429 (222 men, 207 women, mean age 55.18 \pm 14 ) subjects were selected from a consecutive sample at the Cardiovascular Prevention Unit, Pitié-Salpêtrière Hospital, Sorbonne University, Paris, France. Inclusion criteria were: age > 18 years, primary prevention, availability of both retinal arteriole and venule in the AOO image. Exclusion criteria were: secondary prevention for any cardiovascular disease, diabetic and/or hypertensive retinopathy or any known microvascular or macrovascular complication related to HT and/or T2D. The study group was stratified in 4 different subgroups according to normal or high blood pressure (nBP and hBP, respectively, defined according to a cut-off of 140/90 $\mathrm{mmHg}$ for office BP and 135/85 $\mathrm{mmHg}$ for home BP) and/or normal or high fasting plasma glucose (FPG) (nGly and hGly, respectively, defined according to a cut-off of $7 \mathrm{mmol} / \mathrm{L}$ ): $\mathrm{nBP} / \mathrm{nGly}$ subgroup consisted in 154 "control" subjects with no diabetes or sustained HT, 44\% of patients treated for HT with an optimal BP control; hBP/nGly subgroup consisted in 201 uncontrolled hypertensive patients without T2D (126 treated, 75 untreated for HT); nBP/hGly subgroup consisted of 27 subjects without HT and hyperglycaemia; Age $>_{57} / \mathrm{hBP} / \mathrm{hGly}$ subgroup consisted in 47 subjects selected from the upper median age group ( $>57$ years) of the entire population with both uncontrolled BP and hGly. The control subjects were further stratified according to age tertiles in order to analyze age-related changes in retinal microcirculation. 


\section{Measurements (Figure 1)}

Adaptive Optics Camera (AOC) fundus images were obtained using a commercially available flood-illumination AOC retinal camera ( $\mathrm{rtx} 1^{\mathrm{TM}}$; Imagine Eyes, Orsay, France) (20). Image acquisition was performed on the right eye and was started at the optic disc and continued until the first or second branch of the main superior temporal artery to include at least one bifurcation in the acquired field. This region of interest was chosen according to previously published works (15,21-23). Fundus oculi images were acquired including in the same frame both the arteriole and the adjacent venule. If the right eye was not explorable, images were acquired on the left eye. Three adjacent vascular segments were consecutively analysed on a selected $38 \mu \mathrm{m}$ long area for each segment, devoid of arteriovenous nicking or focal arteriolar narrowing. These consecutive measures were performed on the acquired images for both arterioles and venules to obtain the following averaged quantitative measurements: a) arterioles: wall thickness (WT) and internal diameter (aID); b) venules: internal diameter (vID). From these direct measurements the following variables were calculated: arterioles WLR and WCSA as well as arteriovenous ratio (AVR). Furthermore, from the three consecutive measurements of both aID and vID, a coefficient of variation (CV aID and CV vID, respectively) was calculated in order to account for ID irregularity, using the following formula: [(standard deviation (SD) ID/mean ID) x100]. Image processing was performed with the semi-automatic AoDetect ${ }^{\circledR}$ validated software (15). A single experienced operator (AG) performed the image analysis blinded to patients' clinical data. Intra- and interobserver reproducibility of microvascular anatomical indices had been previously assessed $(15,22)$.

\section{Cardiovascular risk factors}

\section{Blood Pressure}

All individuals underwent attended office brachial BP assessment in standard conditions. Office brachial BP was measured simultaneously to AOO assessment after 5 minutes resting in a seated position during the outpatient visit using an oscillometric device (OMRON 905®). Home BP measurements (HBPM) were performed using validated electronic arm devices $\left(\mathrm{OMRON} \AA\right.$, Hartman ${ }^{\circledR}$, Microlife $\left.\AA\right)$. Patients were trained at the physician office on how to perform HBPM: values were recorded as the mean systolic BP (SBP) and diastolic BP (DBP) of 18 measurements ( 3 consecutive measurements made every 5 minutes in the morning and in the evening, after 5 minutes in a seated position, during 3 consecutive days).

Antihypertensive treatments were recorded in all individuals and divided into the following classes: renin angiotensin system (RAS) blockers, calcium channel blockers (CCB), diuretics, beta-blockers (BB) and other class.

\section{Hyperglycaemia.}

Hyperglycaemia was defined by fasting plasma glucose (FPG) levels $\geq 7 \mathrm{mmol} / \mathrm{L}$ and $\mathrm{T} 2 \mathrm{D}$ was defined according to the presence of antidiabetic therapy or two consecutive fasting plasma glucose (FPG) levels $\geq$ $7 \mathrm{mmol} / \mathrm{L}$ or $\mathrm{HbAlc} \geq 6.5 \%$.

\section{Dyslipidaemia and smoking}

Dyslipidaemia was defined as a total serum cholesterol level of $>200 \mathrm{mg} / \mathrm{dL}$, serum LDL cholesterol level of $>116 \mathrm{mg} / \mathrm{dL}$, serum HDL cholesterol level of $<40 \mathrm{mg} / \mathrm{dL}$ for men or $<50 \mathrm{mg} / \mathrm{dL}$ for women, or serum triglycerides level of $>150 \mathrm{mg} / \mathrm{dL}$, or as a daily intake of any lipid-lowering medication.

Current smoking was defined as having smoked at least one cigarette in the last 30 days.

\section{Statistical analysis}

The normal distribution of each variable was assessed through the Kolmogorov-Smirnov test. Parametric continuous variables are reported as mean $+/$ - standard deviation, non-parametric variables are reported as median and interquartile range [Q1; Q3]. Qualitative variables are reported as frequency. 
Comparison of continuous variables across subgroups was performed using One-way ANOVA for parametric variables, and Kruskal-Wallis test for non-parametric variables. Chi-squared test was applied for comparisons among qualitative variables. Univariate regressions were performed with Pearson's correlation test (Spearman's test for non-parametric variables). Furthermore, multivariate linear regression model including age, gender(male=1), SBP, DBP, hyperglycaemia (yes=1, no=0), antihypertensive treatment (yes=1, no=0) as covariates was used to identify independent predictors of retinal microvascular remodeling. Non-parametric variables were log-transformed for the multivariate regression analysis.

Statistical analyses were performed using SPSS software (SPSS version 23, Chicago, IL). Statistical tests were bilateral and a $\mathrm{p}$ value $<0.05$ was considered significant.

\section{Results}

Main demographic, clinical and biochemical characteristics are summarized in Table 1 for the four study subgroups. Except for the age ${ }_{57} / \mathrm{hBP} / \mathrm{hGly}$ group, mean age did not differ among groups. Consistently with the study design, groups with hBP showed higher office and home BP levels, and presence of hGly was associated with higher FPG, HbAlc and triglycerides levels. Subjects from the Age ${ }_{57} / \mathrm{hBP} / \mathrm{hGly}$ group exhibited lower lipid levels, being more treated than the other groups. When considering the entire study group, men exhibited higher creatinine, FPG and triglycerides compared to women (Additional file 1). Women had an increased arteriolar WT and WCSA as compared to men, who had a higher vID resulting in a smaller AVR as compared to women (Additional file 1).

\section{Retinal microvascular remodeling}

Figure 2 shows the boxplots of retinal microvascular measures stratified according to study groups and age tertiles for the control group. The detailed results of AOO findings are shown in Table 2 (Additional file 2).

Effect of ageing. We found a significant progressive increase in WT across age tertiles, resulting in an increase in WLR and WCSA. In addition, no changes in the aID were observed reflecting an euluminal vascular growth. Subjects clinical characteristics of age tertiles are reported in Table 3: older subjects were more treated for dyslipidemia and had slightly higher BP, FPG and HbAlc levels, whereas younger subjects had better lipid profile.

Effect of hBP. The hBP/nGly group exhibited the highest WLR, the narrowest aID and lowest AVR. Arteriolar WT was significantly increased as compared to controls, while no significant differences were observed for WCSA. These features were a marker of eutrophic inward remodeling.

Effect of hGly. In the nBP/hGly group we observed a similar WLR compared to controls. A concomitant nonsignificant increase in aID and WT as compared to controls was observed resulting in an increase in WCSA, suggesting a hypertrophic outward growth.

Combined effect of age, hBP and hGly. Intermediate levels of aID and AVR were observed in this group, compared to $\mathrm{nBP} / \mathrm{nGly}, \mathrm{hBP} / \mathrm{nGly}$ and $\mathrm{nBP} / \mathrm{hGly}$ groups. However, as compared to controls, we observed an increase in WLR. Besides, such patients had the highest WT and WCSA indicating an overall hypertrophic remodeling and growth.

Figure 3 shows arteriolar changes after stratification according to tertiles of age and SBP or FPG. While WT varied with age in the $1^{\text {st }}$ and $2^{\text {nd }}$ tertile of SBP, no changes were found in the highest age and SBP tertiles, which were associated with the highest WT values (Figure 3A). Similarly, WLR is associated with SBP in the $1^{\text {st }}$ and $2^{\text {nd }}$ age tertiles, whereas the highest tertiles of age and SBP were associated with similarly increased WLR (Figure 3B). On a similar basis, both aID and WCSA varied with age and FPG in the lowest FPG and age tertiles, respectively, showing an interaction of these two variables in the higher tertiles that resulted in no significant changes in aID and WCSA (Figure 3C and 3D).

Predictors of arteriolar remodeling (Table 4) 
Univariate analysis showed that age was significantly associated with WT, aID, WLR and WCSA. Multivariate regression analysis confirmed the independent association of WT, WLR and WCSA but not aID with ageing. Systolic BP was correlated with WT and WLR whereas DBP was inversely associated with aID and AVR and directly correlated with CV aID and WLR in the univariate. These associations were confirmed in the multivariate model, except for WLR as a predictor of SBP. Fasting glycaemia was positively associated with aID, vID and WCSA. The association with vID was lost in the multivariate analysis but AVR was independently associated with hGly.

\section{Discussion}

In this study high resolution and non-invasive adaptive optics is used to investigate retinal microcirculation in humans indicating that during ageing the increase in WLR and WCSA was induced by WT changes, whereas a reduced aID in hBP underlain an increased WLR and an increased aID underlain a higher WCSA in hGly. This suggests that vascular remodeling is associated with changes in BP, while ageing and hGly trigger arteriolar hypertrophy (Table 5).

It has been previously reported that arteriolar remodeling takes place in HT (24-26). The nature of this remodeling has been described through in vitro studies on muscular arterioles from gluteal biopsies. An inward eutrophic remodeling was found in HT, characterized by an arteriolar narrowing due to a chronic vasoconstriction possibly related to an adrenergic overdrive that has been suggested as a factor leading to these vascular changes (27). Furthermore, our present results are in line with previous observations in vivo, which showed such eutrophic remodeling in HT, with age and SBP as independent correlates of increased WLR (22).

If WLR is a well-established marker of vascular remodeling, it might not allow a detailed evaluation of the singular contribution of its numerator and denominator. Both wall and lumen carry different physio-pathological information and merging the two components might lead to inappropriate inferences and conclusions. In our study we observed an increased WLR in HT, which was associated to both a decrease in aID and an increase in WT. Systolic BP is the expression of cardiac output and is more strictly related to arterial pulsatility of conductance vessels, whereas DBP represents the peripheral vascular resistance, regulating peripheral blood supply (28). Consequently, SBP varies with changes in arterial compliance, increasing with increasing stiffness (29), and being associated with wall remodeling, whereas DBP changes result from peripheral vascular resistance, which is correlated with vessels calibre. In our study we observed that SBP was an independent predictor of vascular wall, having no effect on arteriolar or venular diameter, while DBP was inversely correlated with arteriolar lumen. Antihypertensive treatment did not appear to influence retinal microvascular remodeling. This is in agreement with previous findings (22), where the effect of BP levels on retinal arteriolar remodeling was independent of antihypertensive treatment. When excluding subjects with ongoing antihypertensive treatment, results did not change (data not shown): patients with a controlled HT under treatment from the control group exhibited same structural features as never treated control subjects: this suggests that a "hypertensive burden" plays a direct role on retinal microcirculation but that this is still reversible. However, the evidence on the effect of treatment on microcirculation needs to be improved $(1,30)$.

Interestingly, arteriolar irregularity, as expressed by the coefficient of variation of arteriolar ID, was the only arteriolar variable not affected by age but associated to DBP levels. This biomarker might be used as a quantitative evaluation of otherwise more qualitative focal arteriolar narrowing (FAN), another microvascular marker of arteriolar remodeling (21) and cerebral or coronary thrombosis (31-33).

The evaluation of the CV ID has been performed on a single vessel and on a limited vascular segment: an underestimation of the presence of these irregularities cannot be excluded as it has not yet been evaluated in the whole arteriolar tree. Nevertheless, this could represent a more valid marker of hypertension in conditions that could mask the effect of HT on retinal arterioles, like T2D.

Rizzoni et al. have confirmed in both in vitro and in vivo studies that in T2D arterioles were found to undergo a hypertrophic remodeling (26), with both an increased media-to-lumen ratio and WCSA. This process may be related to the action of both insulin and insulin-like growth factor-1 that would induce a vasodilation together 
with an increased collagen-elastin ratio (34). Hyperglycaemia itself has been associated with changes in microvascular structure and function, which are mainly mediated by the interaction with perivascular adipocytes (35). In this study we were not able to confirm previous findings of a hypertrophic arteriolar remodeling (26), as WLR was found unchanged as compared to non-diabetic controls. The reason for this discrepancy may be also related to the conditions in which vessels were analysed: our study is a completely non-invasive evaluation of retinal microcirculation, which means that the vessel remodeling is observed under physiological circumstances, whereas previous analyses were performed through a pressure myograph which could not consider all nonpressure stimuli occurring physiologically in microcirculation. Furthermore, retinal microcirculation is a more protected territory where an autoregulation takes place and local metabolic stimuli influence the vascular tone (36), independently from any direct systemic adrenergic drive (37), making the relationship with macrovascular alterations potentially different from other microcirculatory districts. However, an isolated increase in WCSA was confirmed. Previous studies have shown indeed that WLR was not an adequate marker of T2D in early stages of disease $(38,39)$ : this can be easily explained by the harmonic increase in both WT and aID which has no effect on WLR, despite it leads to an increased WCSA. Despite the absence of a different vID in T2D we observed hyperglycaemia to be an independent predictor of AVR. As in the case of WLR, the use of AVR may not consider separate contributions of venular and arteriolar diameters (40). Venular diameter has been associated to vascular changes in T2D and metabolic disorders, although with contrasting results (41-43). In the Multi-Ethnic Study of Atherosclerosis (42) a wider venular calibre was only associated with retinal microvascular changes in diabetic subjects of Hispanic and Chinese origins, whereas no difference was observed in Caucasians, which represent the majority of our population. In another hand, it was reported that wider venular calibres were observed in the presence of diabetic retinopathy (44): this would be an adaptive response to a pathologic arteriolar widening, in order to maintain capillary pressure and consequently protect from target organ damage.

Patients presenting both hBP and hGly had an intermediate WLR level. This finding could be the expression of a much more intricate pathophysiological process taking place in the presence of both HT and T2D: experimental studies have shown that wall shear stress, that depends on blood flow, is associated with changes in microvascular diameter, whereas a circumferential stress, expression of an increased transmural pressure, would influence WT (45). In high BP conditions, the increased transmural pressure may induce an increased circumferential wall stress, resulting in an increased WT, while in T2D an increased blood flow leading to a persistent wall shear stress may determine an increased diameter. As hemodynamic and metabolic stimuli are in mutual relationship both wall circumferential and shear stress could mitigate the increase in WLR, as in the presence of both HT and T2D.

In our study, men had wider veins than women, but this difference was not associated with other clinical findings. Women, however, exhibited an increased WT and WCSA. This difference persisted after adjusting for body surface area (data not shown). This finding may support previous results reporting a higher prevalence of microvascular dysfunction in women as compared to men and possibly linked to specific sex - single nucleotide polymorphism interaction (46), suggesting a possible role of microvascular dysfunction as a leading cause for the higher prevalence of subclinical cardiac disease in women (47).

Dyslipidaemia differed among the study subgroups, with an "atherogenic dyslipidaemia" profile in T2D patients, who therefore had lower LDL-C levels as compared to the non-T2D subgroups. Neither lipid profile nor lipid lowering treatment were shown to influence retinal microvascular remodelling in our analysis (data not shown). However, a more detailed serum apolipoprotein profiling should be performed to better clarify the relationship between retinal microcirculation and lipids. A role of Apolipoprotein-A1, the transporter of HDL-C particles, has been proposed on retinal microvascular function based on the observation of an increased responsiveness to flicker-light stimulation as well as an inverse relationship with vessel tortuosity (48).

Our study presents several limitations: first, this is an observational study and thus unable to distinguish causality from these associations; secondly, the $\mathrm{nBP} / \mathrm{hGly}$ and Age $>57 / \mathrm{hBP} / \mathrm{hGly}$ group samples were small as compared to the $\mathrm{nBP} / \mathrm{nGLy}$ and $\mathrm{hBP} / \mathrm{nGly}$ groups. However, groups were carefully selected in order to avoid an overlap of conditions and potential effects on retinal vascular changes. Finally, we could not perform any functional exploration due to the static nature of the exploration. However, these early morphological changes 
observed through this high-definition technique are very likely the expression of an underlying endothelial dysfunction, making a study with functional testing of high interest.

\section{Conclusions}

Using AOO we characterised in detail retinal microvascular changes in ageing, high blood pressure and hyperglycaemia. We described in an uncomplicated population the different contribution of ageing, blood pressure, as well as of hyperglycaemia, on retinal microcirculation. Adaptive Optics Ophthalmoscopy allows an in-depth analysis of arteriolar wall and calibre leading to a better understanding of the underlying physiopathological processes to target future pharmacological strategies.

\section{Declarations}

\section{Funding}

None

\section{Conflicts of interest/Competing interests (include appropriate disclosures)}

AG declares having received honoraria from Akcea, AMGEN, Mylan, Novartis, Sanofi and Regeneron, Unilever (none of them related to this work); XG declares having received honoraria from Sanofi and Novartis. The remaining authors declare that they have no competing interests.

\section{Availability of data and material (data transparency)}

The datasets used and/or analysed during the current study are available from the corresponding author on reasonable request.

\section{Code availability (software application or custom code)}

Not applicable

\section{Authors' contributions}

AG conceived and designed the research, acquired the data, performed statistical analysis and wrote the first draft of the manuscript. TD performed statistical analysis and contributed to the first draft and critical revision of the manuscript. AGi contributed to data analysis and made critical revision of the manuscript. MP and NK made critical revision of the manuscript for key intellectual content. XG conceived and designed the research, acquired the data, handled funding and supervision and made critical revision of the manuscript for key intellectual content. All authors read and approved the final manuscript

\section{Ethics approval and consent to participate}

Approval of the Ethics Committee of the Saint-Antoine hospital (Paris, France) was obtained. This research was supported by the French Institute of Health and Medical Research (Institut National de la Santé et de la Recherche Médicale, INSERM; No. C10-03 No. IDRCB 2010-A00492-37).

\section{Consent for publication}

All study participants consented to participating in this study, and having their results published as part of this study.

\section{References}


1. Climie Rachel E., van Sloten Thomas T., Bruno Rosa-Maria, Taddei Stefano, Empana Jean-Philippe, Stehouwer Coen D.A., et al. Macrovasculature and Microvasculature at the Crossroads Between Type 2 Diabetes Mellitus and Hypertension. Hypertension. 2019 Jun 1;73(6):1138-49.

2. Strain WD, Paldánius PM. Diabetes, cardiovascular disease and the microcirculation. Cardiovasc Diabetol. 2018 18;17(1):57.

3. Rizzoni D, Rizzoni M, Nardin M, Chiarini G, Agabiti-Rosei C, Aggiusti C, et al. Vascular Aging and Disease of the Small Vessels. High Blood Press Cardiovasc Prev Off J Ital Soc Hypertens. 2019 Jun;26(3):183-9.

4. Wen SW, Wong CHY. Aging- and vascular-related pathologies. Microcirculation. 2019;26(2):e12463.

5. Laurent Stéphane, Boutouyrie Pierre. The Structural Factor of Hypertension. Circ Res. 2015 Mar $13 ; 116(6): 1007-21$.

6. Ikram M. Kamran, Witteman Jacqueline C.M., Vingerling Johannes R., Breteler Monique M.B., Hofman Albert, de Jong Paulus T.V.M. Retinal Vessel Diameters and Risk of Hypertension. Hypertension. 2006 Feb 1;47(2):189-94.

7. Witt Nicholas, Wong Tien Y., Hughes Alun D., Chaturvedi Nish, Klein Barbara E., Evans Richard, et al. Abnormalities of Retinal Microvascular Structure and Risk of Mortality From Ischemic Heart Disease and Stroke. Hypertension. 2006 May 1;47(5):975-81.

8. Sairenchi Toshimi, Iso Hiroyasu, Yamagishi Kazumasa, Irie Fujiko, Okubo Yoshiro, Gunji Junko, et al. Mild Retinopathy Is a Risk Factor for Cardiovascular Mortality in Japanese With and Without Hypertension. Circulation. 2011 Dec 6;124(23):2502-11.

9. Stehouwer CDA. Microvascular Dysfunction and Hyperglycemia: A Vicious Cycle With Widespread Consequences. Diabetes. 2018 Sep;67(9):1729-41.

10. Cheung N, Mitchell P, Wong TY. Diabetic retinopathy. The Lancet. 2010 Jul 10;376(9735):124-36.

11. Antonetti DA, Klein R, Gardner TW. Diabetic Retinopathy. N Engl J Med. 2012 Mar 29;366(13):122739.

12. Wei Y, Jiang H, Shi Y, Qu D, Gregori G, Zheng F, et al. Age-Related Alterations in the Retinal Microvasculature, Microcirculation, and Microstructure. Invest Ophthalmol Vis Sci. 2017 Jul 1;58(9):3804-17.

13. Kotliar KE, Lanzl IM, Hanssen H, Eberhardt K, Vilser W, Halle M, et al. Does Increased Blood Pressure Rather Than Aging Influence Retinal Pulse Wave Velocity? Invest Ophthalmol Vis Sci. 2012 Apr 1;53(4):2119-26.

14. Nagaoka T, Sato E, Takahashi A, Sogawa K, Yokota H, Yoshida A. Effect of aging on retinal circulation in normotensive healthy subjects. Exp Eye Res. 2009 Dec 1;89(6):887-91.

15. De Ciuceis C, Agabiti Rosei C, Caletti S, Trapletti V, Coschignano MA, Tiberio GAM, et al. Comparison between invasive and noninvasive techniques of evaluation of microvascular structural alterations. J Hypertens. 2018;36(5):1154-63.

16. Paques M, Meimon S, Rossant F, Rosenbaum D, Mrejen S, Sennlaub F, et al. Adaptive optics ophthalmoscopy: Application to age-related macular degeneration and vascular diseases. Prog Retin Eye Res. 2018 Sep 1;66:1-16.

17. Schiffrin EL. Vascular remodeling in hypertension: mechanisms and treatment. Hypertens Dallas Tex 1979. 2012 Feb;59(2):367-74. 
18. Bruno Rosa Maria, Grassi Guido, Seravalle Gino, Savoia Carmine, Rizzoni Damiano, Virdis Agostino. Age- and Sex-Specific Reference Values for Media/Lumen Ratio in Small Arteries and Relationship With Risk Factors. Hypertension. 2018 Jun 1;71(6):1193-200.

19. Rosei EA, Rizzoni D. Small artery remodelling in diabetes. J Cell Mol Med. 2010;14(5):1030-6.

20. Lombardo M, Serrao S, Devaney N, Parravano M, Lombardo G. Adaptive optics technology for highresolution retinal imaging. Sensors. 2012 Dec 27;13(1):334-66.

21. Koch E, Rosenbaum D, Brolly A, Sahel J-A, Chaumet-Riffaud P, Girerd X, et al. Morphometric analysis of small arteries in the human retina using adaptive optics imaging. J Hypertens. 2014 Apr 1;32(4):890-8.

22. Rosenbaum D, Mattina A, Koch E, Rossant F, Gallo A, Kachenoura N, et al. Effects of age, blood pressure and antihypertensive treatments on retinal arterioles remodeling assessed by adaptive optics. J Hypertens. 2016 Jun 1;34(6):1115-22.

23. Rosenbaum D, Kachenoura N, Koch E, Paques M, Cluzel P, Redheuil A, et al. Relationships between retinal arteriole anatomy and aortic geometry and function and peripheral resistance in hypertensives. Hypertens Res Off J Jpn Soc Hypertens. 2016 Jul;39(7):536-42.

24. Heagerty AM, Aalkjaer C, Bund SJ, Korsgaard N, Mulvany MJ. Small artery structure in hypertension. Dual processes of remodeling and growth. Hypertens Dallas Tex 1979. 1993 Apr;21(4):391-7.

25. Levy BI, Ambrosio G, Pries AR, Struijker-Boudier HA. Microcirculation in hypertension: a new target for treatment? Circulation. $2001 \mathrm{Aug} 7 ; 104(6): 735-40$.

26. Rizzoni D, Rosei EA. Small artery remodeling in hypertension and diabetes. Curr Hypertens Rep. 2006 Jan 1;8(1):90-5.

27. Porteri E, Rizzoni D, Mulvany MJ, De Ciuceis C, Sleiman I, Boari GE, et al. Adrenergic mechanisms and remodeling of subcutaneous small resistance arteries in humans. J Hypertens. 2003 Dec;21(12):2345-52.

28. Barral J-P, Croibier A. 2 - Circulatory physiology. In: Barral J-P, Croibier A, editors. Visceral Vascular Manipulations [Internet]. Oxford: Churchill Livingstone; 2011 [cited 2019 Jul 8]. p. 27-45. Available from: http://www.sciencedirect.com/science/article/pii/B9780702043512000028

29. Laurent Stéphane, Briet Marie, Boutouyrie Pierre. Large and Small Artery Cross-Talk and Recent Morbidity-Mortality Trials in Hypertension. Hypertension. 2009 Aug 1;54(2):388-92.

30. Wolf M, Ewen S, Mahfoud F, Böhm M. Hypertension: history and development of established and novel treatments. Clin Res Cardiol Off J Ger Card Soc. 2018 Aug;107(Suppl 2):16-29.

31. Baker Michelle L., Hand Peter J., Wang Jie Jin, Wong Tien Y. Retinal Signs and Stroke. Stroke. 2008 Apr 1;39(4):1371-9.

32. Wong TY, McIntosh R. Systemic associations of retinal microvascular signs: a review of recent population-based studies. Ophthalmic Physiol Opt. 2005;25(3):195-204.

33. Heringa SM, Bouvy WH, van den Berg E, Moll AC, Kappelle LJ, Biessels GJ. Associations between retinal microvascular changes and dementia, cognitive functioning, and brain imaging abnormalities: a systematic review. J Cereb Blood Flow Metab Off J Int Soc Cereb Blood Flow Metab. 2013 Jul;33(7):983-95.

34. Rizzoni D, Porteri E, Guelfi D, Muiesan ML, Valentini U, Cimino A, et al. Structural alterations in subcutaneous small arteries of normotensive and hypertensive patients with non-insulin-dependent diabetes mellitus. Circulation. 2001 Mar 6;103(9):1238-44.

35. Bakker W, Eringa EC, Sipkema P, van Hinsbergh VWM. Endothelial dysfunction and diabetes: roles of hyperglycemia, impaired insulin signaling and obesity. Cell Tissue Res. 2009 Jan;335(1):165-89. 
36. Patton N, Aslam T, Macgillivray T, Pattie A, Deary IJ, Dhillon B. Retinal vascular image analysis as a potential screening tool for cerebrovascular disease: a rationale based on homology between cerebral and retinal microvasculatures. J Anat. 2005 Apr;206(4):319-48.

37. Laties AM. Central retinal artery innervation. Absence of adrenergic innervation to the intraocular branches. Arch Ophthalmol Chic Ill 1960. 1967 Mar;77(3):405-9.

38. Jumar A, Ott C, Kistner I, Friedrich S, Michelson G, Harazny JM, et al. Early Signs of End-Organ Damage in Retinal Arterioles in Patients with Type 2 Diabetes Compared to Hypertensive Patients. Microcirculation. 2016;23(6):447-55.

39. Kannenkeril D, Bosch A, Harazny J, Karg M, Jung S, Ott C, et al. Early vascular parameters in the microand macrocirculation in type 2 diabetes. Cardiovasc Diabetol [Internet]. 2018 Sep 19 [cited 2020 Jan 15];17. Available from: https://www.ncbi.nlm.nih.gov/pmc/articles/PMC6146516/

40. Liew G, Sharrett AR, Kronmal R, Klein R, Wong TY, Mitchell P, et al. Measurement of Retinal Vascular Caliber: Issues and Alternatives to Using the Arteriole to Venule Ratio. Invest Ophthalmol Vis Sci. 2007 Jan 1;48(1):52-7.

41. Nguyen TT, Wang JJ, Islam FMA, Mitchell P, Tapp RJ, Zimmet PZ, et al. Retinal Arteriolar Narrowing Predicts Incidence of Diabetes: The Australian Diabetes, Obesity and Lifestyle (AusDiab) Study. Diabetes. 2008 Mar 1;57(3):536-9.

42. Nguyen TT, Wang JJ, Sharrett AR, Islam FMA, Klein R, Klein BEK, et al. Relationship of Retinal Vascular Caliber With Diabetes and Retinopathy: The Multi-Ethnic Study of Atherosclerosis (MESA). Diabetes Care. 2008 Mar 1;31(3):544-9.

43. Mutlu Unal, Ikram M. Kamran, Wolters Frank J., Hofman Albert, Klaver Caroline C.W., Ikram M. Arfan. Retinal Microvasculature Is Associated With Long-Term Survival in the General Adult Dutch Population. Hypertension. 2016 Feb 1;67(2):281-7.

44. Klein R, Klein BEK, Moss SE, Wong TY. Retinal vessel caliber and microvascular and macrovascular disease in type 2 diabetes: XXI: the Wisconsin Epidemiologic Study of Diabetic Retinopathy. Ophthalmology. 2007 Oct;114(10):1884-92.

45. Pries Axel R., Reglin Bettina, Secomb Timothy W. Remodeling of Blood Vessels. Hypertension. 2005 Oct 1;46(4):725-31.

46. Leopold JA. Microvascular dysfunction: genetic polymorphisms suggest sex-specific differences in disease phenotype. Coron Artery Dis. 2014 Jun;25(4):275-6.

47. Halland H, Lønnebakken MT, Pristaj N, Saeed S, Midtbø H, Einarsen E, et al. Sex differences in subclinical cardiac disease in overweight and obesity (the FATCOR study). Nutr Metab Cardiovasc Dis NMCD. 2018;28(10):1054-60.

48. Sasongko MB, Wong TY, Nguyen TT, Kawasaki R, Jenkins AJ, Shaw J, et al. Serum apolipoproteins are associated with systemic and retinal microvascular function in people with diabetes. Diabetes. 2012 Jul;61(7):1785-92. 


\section{Figure legends}

Fig. 1 Schematic representation of direct and calculated measurements performed and obtained through Adaptive Optics Ophthalmoscopy. On the left. Three consecutive measurements were obtained along the same segment of the retinal arteriole and vein. Image acquisition of both vessels was done according to a predefined protocol. The calculation is semi-automatic, in case of obsolete automatic calculation the operator can move the cursor towards the most fitting gradient peak. $\Delta$ stands for changes in internal arteriolar and venular diameter. On the right, image of the retinal vessel segmentation. A, arteriole; V, venule

Fig. 2 Retinal microvascular parameters in the study population according to hBP, hGly and age tertiles. The $\mathrm{p}$ value reported in the upper-right corner of each graph refers to the across-groups analysis. The control group, in the square, was stratified according to age tertiles in order to study the effects of ageing in the absence of hBP and hGly. Age tertiles were defined according to the following age cut-offs: $1^{\text {st }}$ tertile $<47$ years, $2^{\text {nd }}$ tertile 48-60 years, $3^{\text {rd }}$ tertile $>60$ years. The $\mathrm{p}$ value reported in the upper-left corner of each figure refers to the across-tertiles analysis. nBP, normal Blood Pressure; nGly, normal Glycaemia; hBP, high Blood Pressure; hGly, hyperglycaemia

Fig. 3 Distribution of retinal microvascular parameter according to age, SBP and FPG tertiles. Panel A. WT distribution according to tertiles of age and SBP: patients in the highest tertile of age had thicker arteriolar wall independent of SBP. Panel B. WLR distribution according to age and SBP tertiles: SBP influenced WLR only in the $1^{\text {st }}$ and $2^{\text {nd }}$ tertiles of age. Panel C. aID distribution according to tertile of age and FPG: both age and FPG were associated with a higher aID in the $1^{\text {st }}$ age and FPG tertiles. Panel D. WCSA distribution according to tertiles of age and FPG: increasing levels of FPG were related to increased WCSA in the $1^{\text {st }}$ age tertile; likewise, age increased WCSA in the $1^{\text {st }}$ FPG tertile. ${ }^{*} \mathrm{p}<0.05 ; \uparrow \mathrm{p}<0.01 ;+\mathrm{p}<0.001$ across tertiles. SBP, Systolic Blood Pressure; FPG, Fasting Plasma 
Table 1. Study population main characteristics.

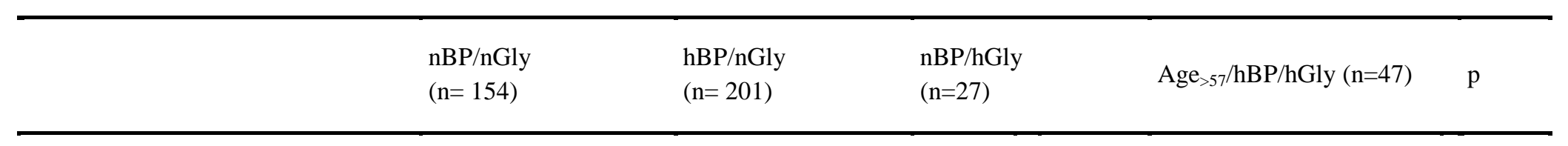

\section{Demographics}

Age, years

Gender, $n$ men / $n$ women

BMI, $K g / m^{2}$

Smoking, $n(\%)$

$$
\begin{aligned}
& 53.5[44 ; 62.3] \\
& 82 / 72 \\
& 25.7[23.0 ; 30.0] \\
& 26(16.9)
\end{aligned}
$$

\section{Blood Pressure}

Office SBP, $m m H g$

Office DBP, $m m H g$

Home SBP, $m m H g$

Home DBP, $\mathrm{mmHg}$

\section{Biochemistry}

Fasting Plasma Glucose, mmol/l

HbA1c, \%

Plasma Creatinine, $\mu \mathrm{mol} / \mathrm{l}$

Total Cholesterol, $m g / d l$

Plasma Triglycerides, $m g / d l$

HDL-cholesterol, $m g / d l$

LDL-cholesterol, $m g / d l$

Antihypertensive treatment

RAS inhibitors, $n(\%)$

Calcium channel blockers, $n(\%)$

$\begin{array}{ccc}53.5[44 ; 62.3] & 55.5[46 ; 68]^{*} & 53.0[45 ; 66] \\ 82 / 72 & 94 / 107 & 14 / 13 \\ 25.7[23.0 ; 30.0] & 26.4[23.5 ; 30.8] & 29.8[25.7 ; 32.7] \dagger \\ 26(16.9) & 31(15.4) & 5(18.5) \\ & & \\ 120.0[112 ; 129] & 148.0[138 ; 158] \dagger & 122.0[113 ; 126] \\ 67.0[61 ; 75] & 80.0[72 ; 92] \dagger & 70.0[63 ; 74] \\ 119.0[112.3 ; 125.0] & 138.1[129.2 ; 148.6] \dagger & 121.0[117.5 ; 127.0] \\ 72.0[65.0 ; 78.4] & 83.4[75.7 ; 93.8] \dagger & 73.0[65.0 ; 76.5]\end{array}$

$64.0[56 ; 69] \dagger \quad<0.001$

$32 / 15 \quad 0.067$

$30.4[28.0 ; 32.6] \dagger \quad<0.001$

$4(8.5) \quad 0.502$

$\begin{array}{cc}149 .[140 ; 159] \dagger & <0.001 \\ 74.0[68 ; 82] \dagger & <0.001 \\ 139.7[129.8 ; 150.2] \dagger & <0.001 \\ 78.0[71.8 ; 84.9] \dagger & <0.001\end{array}$

$\begin{array}{ccc}5.0[4.7 ; 5.5] & 5.1[4.8 ; 5.6] & 8.4[7.6 ; 10.0] \dagger \\ 5.6[5.4 ; 5.8] & 5.7[5.4 ; 5.9] & 7.8[6.9 ; 8.5] \dagger \\ 77.0[65.0 ; 90.0] & 75.0[66.0 ; 81.0] & 72.0[60.0 ; 81.0]\end{array}$

$8.8[7.6 ; 10.4] \dagger$

$<0.001$

$7.6[7.0 ; 8.5] \dagger \quad<0.001$

$85.0[69.0 ; 96.0] \quad 0.037$

$197.0[170.1 ; 239.0] \quad 204.0[174.0 ; 233.0] \quad 196.0[166.0 ; 220.0] \quad 188.0[157.0 ; 204.0]^{*} \quad 0.023$

$94.0[70.0 ; 146.5] \quad 97.0[74.0 ; 146.0] \quad 119.0[87.0 ; 182.0]^{*} \quad 140.0[96.0 ; 262.0] \dagger \quad<0.001$

$50.0[40.5 ; 65.0] \quad 53.0[41.0 ; 67.0] \quad 48.5[36.0 ; 63.0] \quad 40.0[33.0 ; 53.5] \dagger \quad 0.001$

$118.0[98.0 ; 150.0] \quad 125.0[102.0 ; 150.0] \quad 119.0[91.0 ; 139.0] \quad 102.0[82.0 ; 129.0] \$ \quad 0.003$

$\begin{array}{ccccc}67(43.5) & 126(62.7) & - & 32(68.1) & <0.001 \\ 46(29.9) & 89(44.3) & - & 28(59.6) & <0.001 \\ 35(22.7) & 70(34.8) & - & 16(34.0) & 0.001\end{array}$


Diuretics, $n(\%)$

Beta-blockers, $n(\%)$

Antidiabetic treatment

Metformin, $n(\%)$

Sulphanylurea , $n(\%)$

Incretins, $n(\%)$

Insulin, $n(\%)$

Lipid lowering treatment

Statins, $n(\%)$

Ezetimibe, $n(\%)$

Fibrates, $n(\%)$

\section{$24(15.6)$}

$12(7.8)$

$-$

$-$

$-$

$-$

$1(26.6)$

37 (24.0)

$6(3.9)$

3 (1.9)

\section{$50(24.9)$}

40 (19.9)

-

$-$

$-$

$-$

54 (26.9)

47 (23.4)

9 (4.5)

4 (2.0)

-
-
$21(77.8)$
$19(70.4)$
$9(33.3)$
$6(22.2)$
$3(11.1)$
$8(29.6)$
$6(22.2)$
$1(3.7)$
$2(7.4)$

18 (38.3)

16 (34.0)

35 (74.5)

31 (66.0)

18 (38.3)

15 (31.9)

10 (21.3)

24 (51.1)

$21(44.7)$

$3(6.4)$

3 (6.4)
$<0.001$

$<0.001$

$<0.001$

$<0.001$

$<0.001$

$<0.001$

$<0.001$

0.012

0.027

0.920

0.173

${ }^{*} \mathrm{p}<0.05 ; \dagger \mathrm{p}<0.001 ;+\mathrm{p}<0.01$ for comparisons with $\mathrm{nBP} / \mathrm{nGly}$. $\mathrm{nBP}$, normal blood pressure, hBP, high blood pressure; nGly, normoglycaemia; hGly, hyperglycaemia; BMI, body mass index; SBP, systolic blood pressure; DBP, diastolic blood pressure; RAS, renin-angiotensin system. 
Table 2. AOO in the study groups (left) and age tertiles in the control group (right).

\begin{tabular}{|c|c|c|c|c|c|c|c|c|}
\hline $\begin{array}{c}\mathrm{nBP} / \mathrm{nGly} \\
(\mathrm{n}=154)\end{array}$ & $\begin{array}{l}\text { hBP/nGly } \\
(\mathrm{n}=201)\end{array}$ & $\begin{array}{c}\mathrm{nBP} / \mathrm{hGly} \\
(\mathrm{n}=27)\end{array}$ & $\begin{array}{c}\mathrm{Age}_{>57} / \mathrm{hBP} / \mathrm{hGly} \\
(\mathrm{n}=47)\end{array}$ & $\mathrm{p}$ & $\begin{array}{l}\text { Age T1 } \\
(\mathrm{n}=48)\end{array}$ & $\begin{array}{l}\text { Age T2 } \\
(\mathrm{n}=54)\end{array}$ & $\begin{array}{l}\text { Age T3 } \\
(\mathrm{n}=52)\end{array}$ & $\mathrm{p}$ \\
\hline $0.253[0.23 ; 0.29]$ & $0.28[0.25 ; 0.283]^{*}$ & $0.267[0.23 ; 0.29]$ & $0.273[0.253 ; 0.297] \dagger$ & $<0.001$ & $0.247[0.214 ; 0.263]$ & $0.253[0.23 ; 0.288]+$ & $0.263[0.24 ; 0.298] \dagger$ & 0.002 \\
\hline $0.75[0.67 ; 0.83]$ & $0.71[0.62 ; 0.79] \dagger$ & $0.76[0.67 ; 0.88]$ & $0.74[0.69 ; 0.80]$ & 0.012 & $0.76 \pm 0.1$ & $0.753 \pm 0.13$ & $0.746 \pm 0.12$ & 0.695 \\
\hline $23.47[21.4 ; 26.2]$ & $24.87[22.5 ; 27.4] \dagger$ & $24.07[23.4 ; 26.8]$ & $25.9[23.8 ; 28.8]^{*}$ & $<0.001$ & $22.5[20.1 ; 25.2]$ & $23.8[21.8 ; 26.3] \ddagger$ & $24.01[22.5 ; 27.4] \dagger$ & 0.002 \\
\hline $93.6 \pm 11.6$ & $90.2 \pm 13.4 \ddagger$ & $97.7 \pm 12.5$ & $96.1 \pm 12.2$ & 0.002 & $93.9 \pm 11.7$ & $93.4 \pm 11.8$ & $93.6 \pm 11.6$ & 0.922 \\
\hline $2.39[1.6 ; 3.8]$ & $2.69[1.7 ; 4.5]$ & $2.1[1.3 ; 3.8]$ & $2.97[1.6 ; 3.7]$ & 0.352 & $1.99[1.4 ; 3.2]$ & $2.43[1.6 ; 4.0]$ & $2.5[1.7 ; 4.4] \ddagger$ & 0.077 \\
\hline $4011.6 \pm 949$ & $4105.3 \pm 1053$ & $4322.6 \pm 688 t$ & $4524.7 \pm 949 \dagger$ & 0.005 & $3742.3 \pm 814$ & $4011.9 \pm 913$ & $4250.5 \pm 1043 \dagger$ & 0.024 \\
\hline $126.0 \pm 17.3$ & $127.5 \pm 18.9$ & $125.4 \pm 20.5$ & $129.9 \pm 19.9$ & 0.501 & $124.1 \pm 14.6$ & $126.3 \pm 18.9$ & $127.5 \pm 18.0$ & 0.600 \\
\hline $2.67[1.5 ; 4.1]$ & $2.83[1.6 ; 3.9]$ & $3.23[1.9 ; 4.7]$ & $3.1[1.9 ; 4.6]$ & 0.382 & $3.2[1.8 ; 5.0]$ & $2.29[1.2 ; 3.8]$ & $2.6[1.4 ; 4.1]$ & 0.151 \\
\hline
\end{tabular}

* $\mathrm{p}<0.001, \dagger \mathrm{p}<0.01, \ddagger \mathrm{p}<0.05$ versus nBP/nGLy or Age Tertile 1. nBP, normal Blood Pressure, hBP, high Blood Pressure; nGly, normoglycaemia; hGly, hyperglycaemia; WLR, wall-to-lumen ratio; AVR, arterio-venous ratio; WT, wall thickness; aID, arteriolar internal diameter; CV, coefficient of variation; WCSA, wall cross-sectional area; vID, venular internal diameter. 


\begin{tabular}{|c|c|c|c|c|}
\hline & Age Tertile $1(n=48)$ & Age Tertile $2(n=54)$ & Age Tertile $3(n=52)$ & $\mathrm{p}$ \\
\hline \multicolumn{5}{|l|}{ Demographics } \\
\hline Age, years & $36.5 \pm 6.8$ & $52.6 \pm 3.7^{*}$ & $67.1 \pm 5.8^{* \dagger}$ & $<0.001$ \\
\hline Gender $n$ men / $n$ women & $21 / 27$ & $27 / 25$ & $34 / 20$ & 0.148 \\
\hline BMI, $K g / m^{2}$ & $26.9[23.0 ; 33.6]$ & $25.4[23.0 ; 31.6]$ & $25.3[22.5 ; 27.1]$ & 0.174 \\
\hline Smoking, $n(\%)$ & $11(22.9)$ & $8(15.4)$ & $7(13.0)$ & 0.387 \\
\hline \multicolumn{5}{|l|}{ Blood Pressure } \\
\hline Office SBP, $m m H g$ & $116.4 \pm 11.6$ & $118.6 \pm 11.5$ & $122.9 \pm 10.4 \$$ & 0.011 \\
\hline Office DBP, $m m H g$ & $68.9 \pm 10.7$ & $67.6 \pm 10.1$ & $66.4 \pm 9.4$ & 0.486 \\
\hline Home SBP, $m m H g$ & $116.0[106.0 ; 124.4]$ & $118.8[113.6 ; 123.9]$ & $122.5[115.3 ; 126.0] \S$ & 0.091 \\
\hline Home DBP, $m m H g$ & $73.6[65.0 ; 79.9]$ & $72.9[66.0 ; 78.5]$ & $71.3[66.0 ; 77.5]$ & 0.537 \\
\hline \multicolumn{5}{|l|}{ Biochemistry } \\
\hline Fasting plasma glucose, $\mathrm{mmol} / \mathrm{l}$ & $4.7[4.4 ; 5.1]$ & $5.1[4.8 ; 5.5] \S$ & $5.2[5.0 ; 5.6]^{*}$ & 0.001 \\
\hline $\mathrm{HbA1c}, \%$ & $5.6 \pm 0.6$ & $5.7 \pm 0.4 \S$ & $5.7 \pm 0.4 \S$ & 0.043 \\
\hline Plasma Creatinine, $\mu \mathrm{mol} / \mathrm{l}$ & $76.2 \pm 17$ & $78.8 \pm 20$ & $80.9 \pm 16$ & 0.241 \\
\hline Total Cholesterol, $m g / d l$ & $179.0[162.5 ; 204.0]$ & $211.0[168.5 ; 245.8] \S$ & $197.0[174.0 ; 245.0]$ & 0.038 \\
\hline Plasma Triglycerides, $m g / d l$ & $94.0[72.0 ; 135.0]$ & $93.0[65.3 ; 156.8]$ & $95.5[71.0 ; 147.3]$ & 0.994 \\
\hline HDL-cholesterol, $m g / d l$ & $43.0[35.5 ; 52.0]$ & $56.5[41.0 ; 69.0] \ddagger$ & $54.0[45.0 ; 69.5] \ddagger$ & 0.002 \\
\hline LDL-cholesterol, $m g / d l$ & $117.0[96.0 ; 142.0]$ & $130.0[99.0 ; 164.0]$ & $122.0[98.0 ; 156.0]$ & 0.370 \\
\hline \multicolumn{5}{|l|}{ Antihypertensive treatment } \\
\hline RAS inhibitors, $n(\%)$ & $9(18.8)$ & $15(28.8)$ & $22(40.7)$ & 0.058 \\
\hline Calcium channel blockers, $n(\%)$ & $7(14.6)$ & $12(23.1)$ & $16(29.6)$ & 0.213 \\
\hline Diuretics, $n(\%)$ & $6(12.5)$ & $5(9.6)$ & $13(24.1)$ & 0.092 \\
\hline Beta-blockers, $n(\%)$ & $2(4.2)$ & $8(15.4)$ & $2(3.7)$ & 0.05 \\
\hline \multicolumn{5}{|l|}{ Lipid lowering treatment } \\
\hline Statins, $n(\%)$ & $4(8.3)$ & $12(23.1)$ & $21(38.9)$ & 0.002 \\
\hline Ezetimibe, $n(\%)$ & - & $1(1.9)$ & $1(1.9)$ & 0.255 \\
\hline ibrates, $n(\%)$ & $1(2.1)$ & $1(1.9)$ & $1(1.9)$ & 0.994 \\
\hline
\end{tabular}


Table 4. Univariate and multivariate regression analysis for Ageing, hBP and hGly independent determinants.

* $\mathrm{p}<0.001 ; \uparrow \mathrm{p}<0.01 ; \S \mathrm{p}<0.05$ versus Age Tertile $1 ; \dagger \mathrm{p}<0.001$ versus Age Tertile 2 . BMI, body mass index; SBP, systolic blood pressure; DBP, diastolic blor Model A

\begin{tabular}{lcccc|cc} 
& Age & Office SBP & Office DBP & FPG & $\mathbf{R}^{2}$ & Independent correlates \\
\hline WLR & $0.139^{*}$ & $0.220^{*}$ & $0.154^{*}$ & 0.015 & 0.103 & Age*, DBP* \\
WCSA & $0.254^{*}$ & 0.084 & -0.081 & $0.112 \dagger$ & 0.091 & Age*, FPG*, Female gender $\dagger$ \\
WT & $0.252^{*}$ & $0.164^{*}$ & 0.002 & 0.085 & 0.094 & Age*, SBP*, Female gender $\dagger$ \\
aID & $0.106 \dagger$ & -0.087 & $-0.197^{*}$ & $0.150^{*}$ & 0.062 & $\mathrm{DBP}^{*}, \mathrm{FPG}^{*}$ \\
CV aID & -0.018 & 0.064 & $0.215^{*}$ & -0.001 & 0.104 & $\mathrm{DBP}^{*}$
\end{tabular}




\begin{tabular}{|c|c|c|c|c|c|c|}
\hline vID & 0.021 & 0.005 & -0.007 & $0.207^{*}$ & 0.019 & Male gender* \\
\hline AVR & 0.083 & -0.088 & $-.164 *$ & -0.005 & 0.055 & $\mathrm{DBP}^{* *}, \mathrm{FPG} \dagger$, Male gender* \\
\hline
\end{tabular}

Model A: univariate analysis (Pearson's $r$ for parametric variables, Spearmann's rho for non-parametric variables). Model B: multivariate linear regression analysis. Included variables were: Age, SBP, DBP, Hyperglycaemia (yes=1), antihypertensive treatment (yes=1); gender $($ male =1). * $\mathrm{p}<0.001 ; \uparrow \mathrm{p}<0.05$. SBP, Systolic Blood Pressure; DBP, Diastolic Blood Pressure; FPG, Fasting Plasma Glucose; WLR, Wall-to-Lumen Ratio; WCSA; Wall Cross-Sectional Area; WT, Wall Thickness; aID, arteriolar Internal Diameter; CV, coefficient of variation; vID, venular Internal Diameter; AVR, Arterio-Venous Ratio.

Table 5. Graphical representation of arteriolar remodeling/growth according to age, high blood pressure.

\begin{tabular}{c|c|c}
\hline Ageing & Hyperglycaemia & $\begin{array}{c}\text { High Blood Pressure } \\
\text { Age, Hyperglycaemia and High } \\
\text { Blood Pressure }\end{array}$ \\
\hline Euluminal Growth & Outward Growth & $\begin{array}{c}\text { Onward Eutrophic Remodeling } \\
\text { and Growth }\end{array}$ \\
\hline
\end{tabular}




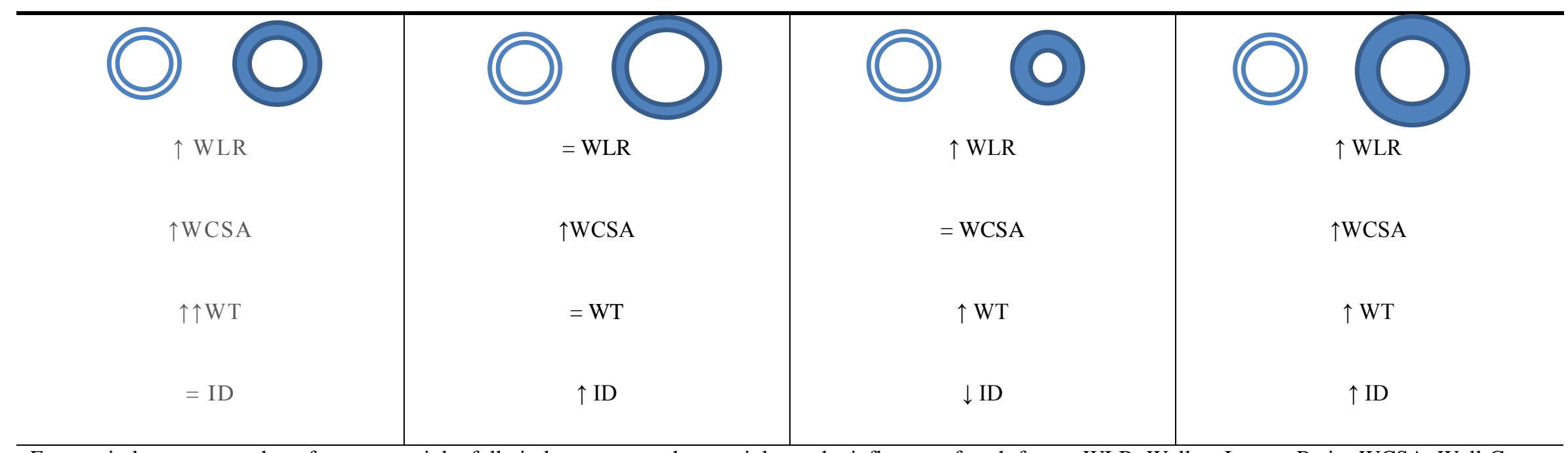

Empty circles represent the reference arteriole, full circles represent the arteriole on the influence of each factor. WLR, Wall-to-Lumen Ratio; WCSA, Wall CrossSectional Area; WT, Wall Thickness; ID, Internal Diameter. 